\title{
Assessment of wild leafy vegetables traditionally consumed by the ethnic communities of Manipur, northeast India
}

\author{
Surjata Konsam ${ }^{1}$ Biseshwori Thongam ${ }^{1 *}$ and Arun Kumar Handique ${ }^{2}$
}

\begin{abstract}
Background: The NE region of India falls in the global hotspot of biodiversity. Wild edible plants (WEPs) are widely consumed in the daily diet of the local people. WEPs are critical for the sustenance of ethnic communities and also as a source of income. However, WEPs received a little attention in research activities, economic development, biodiversity conservation and sustainable management. Many are largely ignored and remained unexplored. With a view of reducing the gap in traditional knowledge and tapping the hidden potential resources for proper utilization, exploitation, and sustainable management of WEPs are crucial.
\end{abstract}

Methods: Surveys were conducted at 20 major markets in all districts of Manipur throughout different seasons from August 2012 to March 2014. A total of 154 avid plant collectors and sellers were interviewed using semi-structured questionnaire, formal, informal and extensive interactions to gather detailed information about these species. An integrated assessment of 68 wild leafy vegetables was also carried out to prioritize them for proper exploitation, conservation, and sustainable management.

Results: A total of 68 wild edible vegetables belonging to 42 families were documented which are being used by indigenous communities for nutritive and therapeutic purposes. Of these species, 54 are perennial (79\%) while others are annual (19\%). Herbaceous plants make up the highest proportion of edible plants. Leaves are dominant edible part followed by shoot and stem, and most are consumed through cooked food. Further, 57 species (84 \%) are commonly available, and 11 (16\%) are rare. According to integrated assessment, 2 species have highest integrated value, 26 species have high value, 31 species have general value and 9 species are of low value. The majority of the species have a high or general value.

Conclusion: Manipur has rich wild vegetable resources. However, many of them are seldom collected or cultivated given their importance in sustaining and diversifying diet. A comprehensive assessment indicated that majority of these plants have high value. Priority species require further research into their nutritional components to understand the potential as a source of future food and nutritional security. They should be promoted for integration into the agricultural system and income generation for local sustenance.

Keywords: Wild edible plants, Local market, Integrated assessment, Sustainability, Food and nutritional security, Manipur

\footnotetext{
* Correspondence: b_thongam07@yahoo.com

${ }^{1}$ Plant Systematics and Conservation Laboratory (PSCL), Institute of

Bioresources and Sustainable Development (IBSD), Takyelpat, Imphal 795001,

Manipur, India

Full list of author information is available at the end of the article
} 


\section{Background}

At present, about $90 \%$ of global food production comes from less than 30 species and more than $85-90 \%$ of total caloric intake obtained from 12 domesticated species [1]. This situation may create an intense biotic and abiotic pressure to the modern agriculture in future. The majority of the edible plants are neglected which grow naturally in the wild and do not have to be tended before producing edible parts [2]. Such edible wild plants can significantly increase sustainability by reducing the risk of over-dependence on a limited number of crops.

The use of wild plants as food is an integral part of the culture and tradition of many indigenous communities around the world. A large section of the rural population meets their nutritional requirement through unconventional means, by consuming various wild plants and animal resources [3]. Millions of people, mostly in developing countries, derive a substantial part of their subsistence and income from wild plant products [4]. WEPs constitute an essential component in the variation of diet and bring household food security of many ethnic communities.

WEPs provide staple food for indigenous people and serve as complementary food for non-indigenous people and offer an alternative source of income [5-7]. They are an important source of nutrient, vitamin and mineral supplements for indigenous population $[8,9]$ and hence, reduce the vulnerability of local communities to food insecurity and thereby act as a buffer for food shortage during the emergency $[10,11]$. Several researchers also demonstrated that many WEPs have nutritional or therapeutic value due to the presence of biologically active compounds, and therefore, can be considered as foodmedicine and quality food $[11,12]$. Many traditional leafy vegetables have higher nutritional values than several known common cultivated plants [13, 14].WEPs have substantial potential to increase the sustainability of agriculture through the reduction in multi-agricultural input. They can also be used for the development of new crops through domestication and benefit modern agriculture by providing plant breeders with a broad pool of potentially useful genetic resources for crop improvement $[15,16]$. The genes for higher productivity and distinctive quality traits may be hidden in this gene pool.

Research on wild food plants is still active even in the present day. Such research is carried out in many countries and continents [17-20]. In the Indian subcontinent, 9500 wild plants are used for food, medicine, fodder, fiber, fuel, essence, cultural and other purposes by over a 53 million tribes belonging to 550 different communities [21]. Ethnobotanical studies on wild food plants associated with tribal communities of central India, Tamil Nadu, Maharashtra, Northeastern India, etc. [21-24] are reported from India. The tribal communities of the Himalayan region of India use over 195 wild edible species [25]. Wild food plants and vegetables being sold in the local markets of South Korea, Croatia, and Turkey [26-28] have also been reported. The local market provides much information on the ethnobotanical process of plant-people interaction and relationships. It represents an intensified interaction between people of different socio-economic groups and specific plants as well [29].

In spite of their immense importance as a valuable food source, WEPs remain widely unknown. Many of the wild food plants are restricted to certain areas or communities. Given the rapid decline of traditional knowledge about WEPs and increased reliance on processed food, documentation and evaluation of the traditional knowledge related to the diversity, usage, and status of WEPs are crucial. Some studies on ethnomedicinal plants have been conducted in Manipur; however, there is limited information on wild vegetables despite its diverse uses. Moreover, information on the nutritional values of most of the WEPs of Manipur is not available. Research and development activities to tap these assets for economic development and sustainability have also remained at the bottom. Many more wild species believed to be edible are yet undocumented. The rich biodiversity of wild plants will be useful in screening newer source of vegetables for present and future need. Inventory of wild food resources, ethnobotanical information on their diversity, usage, status, etc. coupled with nutritional evaluation can establish native species as an alternative to achieve food and nutritional security.

Our objective is to document and assess the diverse wild vegetable resources sold at the local markets of Manipur throughout different seasons. It also aims to provide a systematic way for prioritizing high-quality species through an integrated assessment. It will be utilized further for evaluation of nutritional components of priority species, their integration into the agricultural system and sustainable conservation and management.

\section{Materials and methods Study area}

This study was carried out in Manipur, one of the seven states of Northeast India that forms an integral part of the Indo-Burma biodiversity hotspot. The Manipur state $\left(23^{\circ}\right.$ $27^{\prime}$ to $25^{\circ} 41^{\prime} \mathrm{N}$ latitude and between $93^{\circ} 61^{\prime}$ and $94^{\circ} 48^{\prime} \mathrm{E}$ longitude) comprises an area of $22,327 \mathrm{~km}^{2}$ and administratively divided into 9 districts, of which 4 are valley (viz. Imphal East, Imphal West, Thoubal and Bishnupur) and the rest 5 are hill districts (Chandel, Churachandpur, Senapati, Ukhrul and Tamenglong (Fig. 1). The state is rich in both cultural and biological diversity, having populated by diverse ethnic, linguistic and religious groups including many indigenous tribes. Racially, Manipuri people are unique and have features similar to Southeast Asian. The state has four major ethnic communities - Meitei (Hindu), Naga and Kuki (Tribal communities) and Pangal 


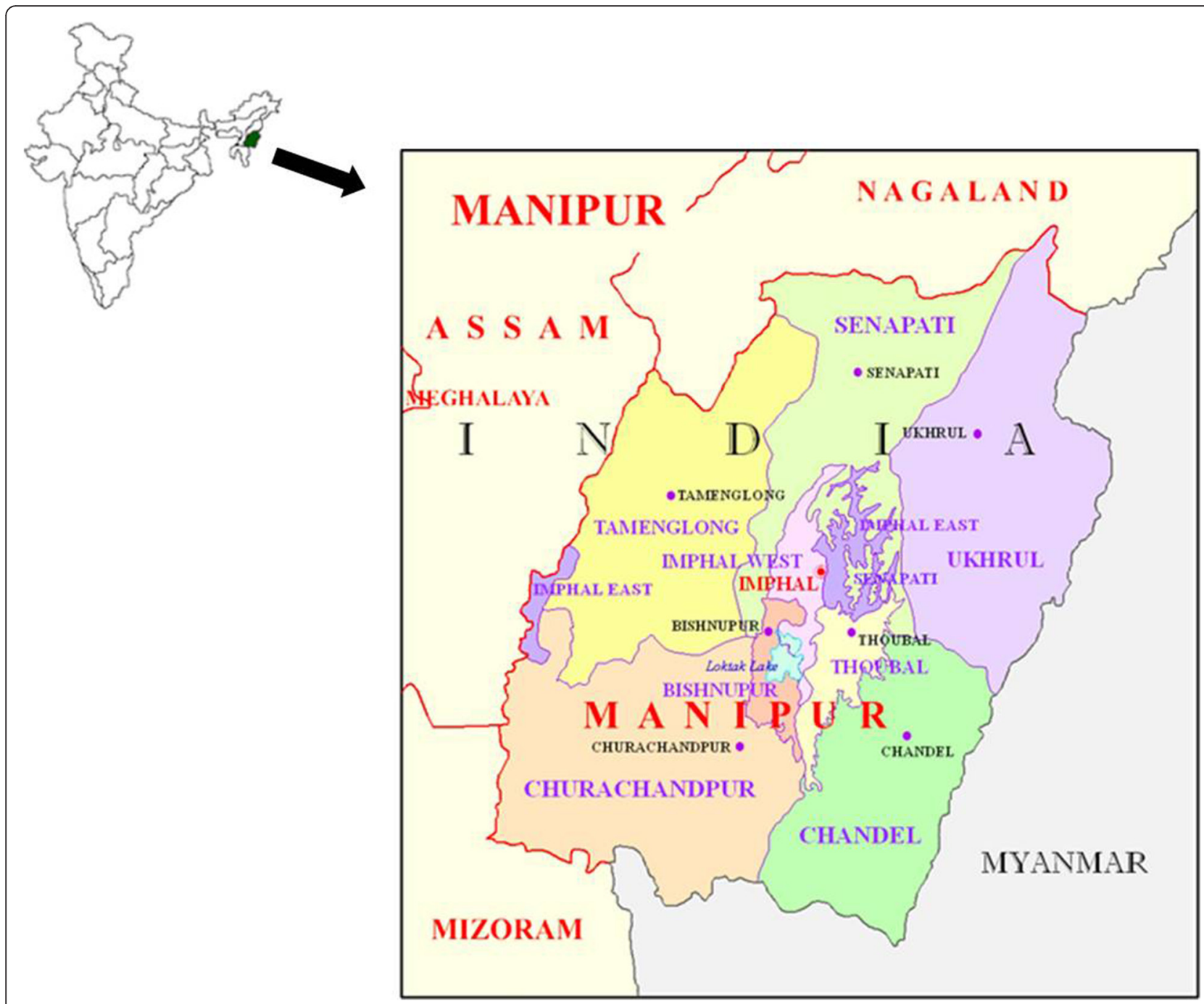

Fig. 1 Location map of study site in Manipur, Northeast India

(Muslim). The Meiteis are the dominant non-tribal community constituting $92 \%$ of the valley area along with the Pangal (minority group), and the five hill districts are inhabited by about 34 ethnic tribes representing $30 \%$ of the state population. They practice distinct culture and tradition and have different socio-economic features. Agriculture is the single largest occupation in Manipur and the mainstay of the state's economy. The trade of wild vegetables provides an alternative source of income and is mainly done by women. Forests account for $67 \%$ of the total land area of this state. The tribal communities collect a large variety of edible and other useful plants from the forest and surrounding wasteland. They also sell a large variety of such plants in the local market. The famous "Ima Keithel" (meaning "Mother's market") of Manipur which sells vegetables and other household items are exclusively run and controlled by women signifying their role in the society both socio-cultural and economically.
A total of 20 major markets were chosen for this study as they form the primary source of supply for wild edible plants in the state -viz 1. Imphal East - Lamlong bazaar and Chingmeirong bazaar 2. Imphal West - Khwairamban keithel and Singjamei bazaar 3. Thoubal District - Thoubal bazaar and Kakching bazaar 4. Bishenpur District - Nambol bazaar, Bishenpur bazaar, Ningthoukhong bazaar and Moirang bazaar 5. Chandel District - Chandel main market and Pallel bazaar 6. Churachandpur District - New Lamka bazaar and Tuibong bazaar 7. Senapati District - Kangpokpi bazaar and Senapati bazaar 8. Ukhrul District - Yaingangpokpi bazaar and Ukhrul main market 9. Tamenglong District - Noney bazaar and Tamenglong bazaar.

\section{Methods}

Survey and data collection

The methods employed in this study were designed for collecting baseline information on the diversity and usage of 
wild vegetable resources locally used by people of Manipur. Before conducting the survey, prior information consent was obtained from the interviewee by explaining the aim of the study. Participants in the study were selected by purposive sampling method. The criterion was to understand and obtain maximum possible information on edibility, medicinal, dietary preference, cultural association and market of wild vegetables from various user communities to come to a generalized inference on WEPs.

Markets were surveyed to assess the presence and abundance of wild edible plants. Detailed studies were conducted at 20 major markets in all districts of Manipur from August 2012 to March 2014 in different season. Each of this market was examined twice in every season between 6.00 and 9.00 am and 2-5 pm. A total of 154 semistructured interviews were carried out with 130 female and 24 male in the age group of 30-77 years for the collection of data. Whenever necessary, translators were used while collecting data as the participants belong to different ethnic communities. However, a majority of them know Manipuri, the state language. Detailed information was gathered using formal, informal and extensive interactions with the wild plant vendor and those involved in the collection and marketing of WEPs following the methods of Upetry et al. [18] and Jain et al. [30]. The inquiries comprise their local names, sources, life forms, growth habit, availability period, edible part, mode of consumption, availability status, distribution pattern, and mode of propagation (Table 1). The collected specimens were identified with the help of experts, relevant literature and Flora [31-34]. The plant nomenclature and author abbreviations follow The Plant List [35]. The specimens were deposited in the Herbarium of Plant Systematics and Conservation Laboratory, Institute of Bioresources and Sustainable Development, Imphal, Manipur.

Further, to perform an integrated assessment of 68 species of wild edible vegetable, the authors used Analytical hierarchy process (AHP) method [36].

\section{Data analysis}

For a systematic approach to integrated assessment, ten evaluation criteria considered important to determine the value of wild edible vegetable were selected, and a score was assigned to each of them (Table 2). These are Taste (T), Distribution (D), Community status (CS), Life form (LF), Basis of civil use (BCU), Wild or cultivated (WC), Edible time (ET), Edible part (EP), Medicinal value (MV), and Market potential (MP).

\section{Weight determination}

Weight determination was based on Analytical hierarchy process (AHP) method [36]. According to the relative importance of each evaluation criteria, the weight of each criterion can be determined. This paper applied the subjective weighting method. The weight of each criterion was calculated using the following steps:

Step1. A hierarchy was constructed based on the ten evaluation criteria viz. Taste (C1), Distribution (C2), Community status (C3), Edible time (C4), Edible part (C5), Life form (C6), Wild or cultivated (C7), Basis of civil use (C8), Medicinal value (C9), and Market potential (C10) with a total of 28 sub-criteria (Table 2).

Step2. This step is to define the relative importance of each criterion by making a pairwise comparison. The seven-point preference scale of Saaty [37] was used as the fundamental scale for this analysis. If two attributes were equally preferred a score of 1 was assigned, judgement moderately favoured one over other - assignment score 3, one strongly favoured over another - assignment score 5 , one very strongly favoured over another - assignment score 7; intermediate values of 2,4,6 were assigned when compromisation needed in decision making. If a criterion was preferred more than the comparison criteria, the reciprocal was assigned to the comparison criteria. The use of reciprocals yields the property that $(a i, j)(a j, i)=1$, where ai, $j$, the preference score of criterion i to criterion j; aj, $i$, preference score of criterion $j$ to criterion $i$ and ai, $j=1 / a j, i$ [38]. Judgement matrix and consistency check of the evaluation model is constructed in Table 3.

Step3. The weights of the decision elements were computed using the eigenvalue $(\lambda \max )$. The consistency index $(\mathrm{CI})$ was computed from the eigenvalue as $\mathrm{CI}=(\lambda \max -\mathrm{n}) /(\mathrm{n}-1)$. The consistency indices of randomly generated reciprocal matrices from the scale 1 to 7 are called the random indices, RI. The RI for matrices of order ' $n$ ' is given in Table 4 [37]. The upper row is the order of the matrix (n), and the lower is the corresponding consistency index of the random judgements. The ratio of 'CI' to 'RI' for the same order matrix is called the consistency ratio (CR), which defines the accuracy of comparisons. The integrated weight of each of the index and the overall weight is then calculated.

Step4. The integrated value (IV) of each species was calculated using the following formula [39] IV $=0.19348 \times$ $\mathrm{T}+0.1920 \times \mathrm{D}+0.0749 \times \mathrm{CS}+0.0933 \times \mathrm{ET}+0.1644 \times \mathrm{EP}$ $+0.0283 \times \mathrm{LF}+0.0358 \times \mathrm{WC}+0.057 \times \mathrm{BCU}+0.0324 \times$ $\mathrm{MV}+0.1275 \times \mathrm{MP}$.

\section{Results and discussion}

\section{Main characteristics and consumption pattern of wild edible plants}

The northeast region of India, a major part of the IndoBurma hotspot, supports considerable biodiversity. The 
Table 1 Names, Life forms, Growth habit, Edible parts, Mode of utilization, Availability period and Availability status

\begin{tabular}{|c|c|c|c|c|c|c|c|c|}
\hline $\begin{array}{l}\text { Local names } \\
\text { (Voucher no.) }\end{array}$ & Scientific names & Family & $\begin{array}{l}\text { Life } \\
\text { forms }\end{array}$ & $\begin{array}{l}\text { Growth } \\
\text { habit }\end{array}$ & Edible part(s) & $\begin{array}{l}\text { Mode of utilization } \\
\text { or preparation }\end{array}$ & $\begin{array}{l}\text { Local availability } \\
\text { period }\end{array}$ & $\begin{array}{l}\text { Local availability status } \\
\text { (in its season) }\end{array}$ \\
\hline $\begin{array}{l}\text { Chuchurangmei IBSD/ } \\
\text { WEP } 001\end{array}$ & Sesbania sesban (L.) Merr & Leguminosae & Annual & Shrub & Fruit, leaf & $\begin{array}{l}\text { Raw leaves added to singju, fruit } \\
\text { cooked eaten as eromba }\end{array}$ & $\begin{array}{l}\text { August- } \\
\text { September }\end{array}$ & Common \\
\hline Kolamni IBSD/WEP 002 & Ipomoea aquatica Forssk & Convolvulaceae & Perennial & Herb & Stem & Cooked eaten as vegetable & Year round & Common \\
\hline $\begin{array}{l}\text { Komprek IBSD/WEP } \\
003\end{array}$ & $\begin{array}{l}\text { Oenanthe javanica (Blume) } \\
\text { DC. }\end{array}$ & Apiaceae & Perennial & Herb & Leaf, stem & $\begin{array}{l}\text { Eaten raw in singju or cooked as } \\
\text { mixed vegetable }\end{array}$ & Year round & Common \\
\hline $\begin{array}{l}\text { Sinjupaal IBSD/WEP } \\
004\end{array}$ & $\begin{array}{l}\text { Alocasia cucullata (Lour.) } \\
\text { G. Don }\end{array}$ & Araceae & Perennial & Herb & Corm & $\begin{array}{l}\text { Eaten raw as singju or cooked with } \\
\text { potato and dry fish }\end{array}$ & Year round & Common \\
\hline YelangIBSD/WEP 005 & Polygonum barbatum L. & Polygonaceae & Annual & Herb & Leaf & Cooked eaten as vegetable & January-March & Common \\
\hline $\begin{array}{l}\text { Kakthum IBSD/WEP } \\
006\end{array}$ & $\begin{array}{l}\text { Eleocharis dulcis (Burm.f.) } \\
\text { Trin. ex Hensch }\end{array}$ & Cyperaceae & Perennial & Herb & Root & $\begin{array}{l}\text { Eaten raw or steam as snack, also } \\
\text { cooked eaten as eromba }\end{array}$ & $\begin{array}{l}\text { November- } \\
\text { December }\end{array}$ & Common \\
\hline $\begin{array}{l}\text { Ching yensil } \\
\text { IBSD/WEP } 007\end{array}$ & $\begin{array}{l}\text { Antidesma diandrum } \\
\text { (Roxb.) B.Heyne. ex Roth }\end{array}$ & Euphorbiaceae & Perennial & Small tree & Leaf & $\begin{array}{l}\text { Cooked eaten as eromba or with } \\
\text { potato and dry fish }\end{array}$ & April -July & Common \\
\hline KengoilBSD/WEP 008 & $\begin{array}{l}\text { Lysimachia ovovata } \\
\text { Buch.-Ham. ex Wall }\end{array}$ & Primulaceae & Perennial & Herb & Whole part & $\begin{array}{l}\text { Cooked eaten as eromba, or with } \\
\text { potatoes and dry fish }\end{array}$ & Winter & Common \\
\hline PerukIBSD/WEP 009 & Centella asiatica (L.) Urb. & Apiaceae & Perennial & Creeper & Whole plant & $\begin{array}{l}\text { Boil eaten (champhut), or with potato } \\
\text { and smashed with chilli, fermented } \\
\text { fish (kangsu) }\end{array}$ & Year round & Common \\
\hline Thamou IBSD/WEP 010 & Nelumbo nucifera Gaertn. & Nelumbonaceae & Perennial & $\begin{array}{l}\text { Rooted } \\
\text { hydrophyte }\end{array}$ & Leaf, root & $\begin{array}{l}\text { Eaten raw snack or singju; cooked } \\
\text { with honey }\end{array}$ & June- October & Common \\
\hline TharoIBSD/WEP 011 & Nymphaea nouchali Burm.f. & Nymphaeaceae & Perennial & $\begin{array}{l}\text { Rooted } \\
\text { hydrophyte }\end{array}$ & Stem, tuber & $\begin{array}{l}\text { Eaten raw - singju; boiled tuber eaten } \\
\text { as snack }\end{array}$ & July- October & Common \\
\hline $\begin{array}{l}\text { Thangjing IBSD/WEP } \\
012\end{array}$ & Euryale ferox Salisb. & Nymphaeaceae & Annual & $\begin{array}{l}\text { Rooted } \\
\text { herb }\end{array}$ & Seed, stem & $\begin{array}{l}\text { Eaten raw mixing with chilli and } \\
\text { fermented fish or singju, added } \\
\text { to cooked dish }\end{array}$ & June-September & Common \\
\hline $\begin{array}{l}\text { Esing ekaithabi } \\
\text { IBSD/WEP } 013\end{array}$ & Neptunia oleracea Lour. & Leguminosae & Annual & Herb & Shoot & $\begin{array}{l}\text { Raw as singju, cooked with other } \\
\text { vegetables }\end{array}$ & Rainy season & Common \\
\hline Koukha IBSD/WEP 014 & Sagittaria sagittifolia $\mathrm{L}$. & Alismataceae & Perennial & Herb & Tuber & $\begin{array}{l}\text { Cooked eaten as eromba or fried } \\
\text { with gram flour }\end{array}$ & $\begin{array}{l}\text { November- } \\
\text { January }\end{array}$ & Common \\
\hline $\begin{array}{l}\text { Yendang IBSD/WEP } \\
015\end{array}$ & Cycas pectinata Buch.-Ham & Cycadaceae & Perennial & Shrub & Leaf, shoot & $\begin{array}{l}\text { Raw as singju or cooked eaten as } \\
\text { eromba }\end{array}$ & June-September & Uncommon \\
\hline $\begin{array}{l}\text { Monsaobi IBSD/WEP } \\
016\end{array}$ & Chenopodium album $\mathrm{L}$. & Amaranthaceae & Annual & Herb & Leaf & Cooked eaten with other vegetables & June-September & Common \\
\hline $\begin{array}{l}\text { Kanghumaan } \\
\text { IBSD/WEP } 017\end{array}$ & $\begin{array}{l}\text { Meriandra bengalensis } \\
\text { (Roxb.) Benth. }\end{array}$ & Lamiaceae & Perennial & Shrub & $\begin{array}{l}\text { Inflorescence, } \\
\text { leaf }\end{array}$ & $\begin{array}{l}\text { Added raw as dressing in eromba } \\
\text { or singju }\end{array}$ & $\begin{array}{l}\text { November- } \\
\text { March }\end{array}$ & Common \\
\hline Tekta IBSD/WEP 018 & $\begin{array}{l}\text { Pogostemon purpurascens } \\
\text { Dalzell }\end{array}$ & Lamiaceae & Annual & Shrub & Leaf & Added as spices & $\begin{array}{l}\text { September- } \\
\text { October }\end{array}$ & Uncommon \\
\hline $\begin{array}{l}\text { Yerum keirum } \\
\text { BSD/WEP } 019\end{array}$ & Stellaria media (L.) Vill. & Caryophyllaceae & Annual & Herb & Whole plant & Cooked as vegetable & Winter season & Common \\
\hline
\end{tabular}


Table 1 Names, Life forms, Growth habit, Edible parts, Mode of utilization, Availability period and Availability status (Continued)

\begin{tabular}{|c|c|c|c|c|c|c|c|c|}
\hline $\begin{array}{l}\text { Toninkhok IBSDMEP } \\
020\end{array}$ & Houttuynia cordata Thunb. & Saururaceae & Perennial & Herb & Whole plant & Use as spice or accessory additives & Year round & Common \\
\hline Loklei IBSD/WEP 021 & $\begin{array}{l}\text { Hedychium coronarium } \\
\text { J. Koenig }\end{array}$ & Zingiberaceae & Perennial & Herb & Rhizome & Boiled eaten as eromba & April-May & Common \\
\hline Pullei IBSD/WEP 022 & Alpinia nigra (Gaertn.) Burtt & Zingiberaceae & Perennial & Herb & Rhizome & Boiled eaten as eromba & April- July & Common \\
\hline NamralBSD/WEP 023 & Amomum aromaticum Roxb. & Zingiberaceae & Perennial & Herb & Stem & Boiled eaten as eromba & $\begin{array}{l}\text { April- } \\
\text { September }\end{array}$ & Common \\
\hline Yaipal IBSD/WEP 024 & Curcuma angustifoliaRoxb. & Zingiberaceae & Perennial & Herb & Inflorescence & $\begin{array}{l}\text { Boiled eaten as eromba, cooked, } \\
\text { as well as fry eaten }\end{array}$ & April- May & Common \\
\hline $\begin{array}{l}\text { Sarei mapan } \\
\text { IBSD/WEP } 025\end{array}$ & Amomum sp. & Zingiberaceae & Perennial & Herb & Inflorescence & $\begin{array}{l}\text { Cooked eaten as eromba and as } \\
\text { mixed vegetable fry }\end{array}$ & February-May & Uncommon \\
\hline $\begin{array}{l}\text { Esing kambong } \\
\text { IBSD/WEP } 026\end{array}$ & $\begin{array}{l}\text { Zizania latifolia (Griseb.) Turcz. } \\
\text { ex Stapf }\end{array}$ & Poaceae & Perennial & Herb & Culms & $\begin{array}{l}\text { Raw-snack, roast, cook as vegetables, } \\
\text { cook with milk honey and black rice }\end{array}$ & $\begin{array}{l}\text { September- } \\
\text { November }\end{array}$ & Uncommon \\
\hline $\begin{array}{l}\text { Chantruk mana } \\
\text { IBSD/WEP } 027\end{array}$ & Cardamine hirsuta $\mathrm{L}$. & Brassicaceae & Annual & Herb & Leaf & $\begin{array}{l}\text { Added raw in singju, additives in } \\
\text { cooked curry }\end{array}$ & $\begin{array}{l}\text { November- } \\
\text { February }\end{array}$ & Common \\
\hline $\begin{array}{l}\text { *Huikhong/mansam } \\
\text { IBSD/WEP } 028\end{array}$ & Viola pilosa Blume & Violaceae & Perennial & Creeper & Whole plant & $\begin{array}{l}\text { Added raw in singju, cooked eaten } \\
\text { as eromba and kangsu }\end{array}$ & $\begin{array}{l}\text { Year round; } \\
\text { except winter }\end{array}$ & Uncommon \\
\hline Phunil IBSD/WEP 029 & Gnaphalium indicum L. & Asteraceae & Annual & Herb & Whole part & $\begin{array}{l}\text { Eaten raw as dressing, cooked eaten } \\
\text { as vegetable }\end{array}$ & $\begin{array}{l}\text { October- } \\
\text { December }\end{array}$ & Common \\
\hline $\begin{array}{l}\text { Kongouyen } \\
\text { IBSD/WEP } 030\end{array}$ & Cissus javanica DC & Vitaceae & Perennial & Climber & Leaf, stem & $\begin{array}{l}\text { Cooked by boiling with potatoes } \\
\text { and dry fish }\end{array}$ & Rainy season & Common \\
\hline $\begin{array}{l}\text { Heibi mana } \\
\text { IBSD/WEP } 031\end{array}$ & $\begin{array}{l}\text { Vangueria spinosa (Roxb. } \\
\text { ex Link) Roxb. }\end{array}$ & Rubiaceae & Perennial & Tree & Leaf & Added raw in singju & Year round & Common \\
\hline $\begin{array}{l}\text { Lam khamen } \\
\text { IBSD/WEP } 032\end{array}$ & Solanum torvum Sw. & Solanaceae & Perennial & Shrub & Fruit & Cooked eaten as vegetable & July-September & Common \\
\hline $\begin{array}{l}\text { Nongmangkha } \\
\text { IBSD/WEP } 033\end{array}$ & $\begin{array}{l}\text { Phlogacanthus thyrsiformis } \\
\text { (Roxb. ex Hardw.) Mabb }\end{array}$ & Acanthaceae & Perennial & Shrub & Inflorescence & $\begin{array}{l}\text { Eaten raw with chutney, cooked } \\
\text { with other vegetable }\end{array}$ & $\begin{array}{l}\text { December- } \\
\text { March }\end{array}$ & Common \\
\hline $\begin{array}{l}\text { Oosingsha mapaan } \\
\text { IBSD/WEP } 034\end{array}$ & Litsea cubeba (Lour.) Pers. & Lauraceae & Perennial & Tree & $\begin{array}{l}\text { Inflorescence, } \\
\text { fruit }\end{array}$ & $\begin{array}{l}\text { Eaten raw with chutney, cooked as } \\
\text { eromba, }\end{array}$ & November-April & Common \\
\hline $\begin{array}{l}\text { Chigonglei angouba } \\
\text { IBSD/WEP } 035\end{array}$ & $\begin{array}{l}\text { Leucaena leucocephala (Lam.) } \\
\text { de Wit }\end{array}$ & Leguminosae & Perennial & Tree & Fruit & Eaten raw as singju, cooked as eromba & $\begin{array}{l}\text { October- } \\
\text { December }\end{array}$ & Common \\
\hline Oothum IBSD/WEP 036 & $\begin{array}{l}\text { Wendlandia paniculata (Roxb.) } \\
\text { DC }\end{array}$ & Rubiaceae & Perennial & Tree & Tender leaf & $\begin{array}{l}\text { Cooked eaten as eromba with black } \\
\text { pea or making chutney }\end{array}$ & March-April & Common \\
\hline $\begin{array}{l}\text { Mukthrubi IBSD/WEP } \\
037\end{array}$ & $\begin{array}{l}\text { Zanthoxylum acanthopodium } \\
\text { DC. }\end{array}$ & Rutaceae & Perennial & Tree & $\begin{array}{l}\text { Leaf, } \\
\text { inflorescence }\end{array}$ & $\begin{array}{l}\text { Eaten raw with chili and fermented } \\
\text { fish chutney or additives in snail curry }\end{array}$ & Year Round & Common \\
\hline $\begin{array}{l}\text { Naoseknambi } \\
\text { IBSD/WEP } 038\end{array}$ & Zanthoxylum sp. & Rutaceae & Perennial & Tree & Leaf & $\begin{array}{l}\text { Raw-singju, added in meat curry, } \\
\text { cooked eaten as kangsoi }\end{array}$ & April-June & Common \\
\hline $\begin{array}{l}\text { Awaphadigom } \\
\text { IBSD/WEP } 039\end{array}$ & Eryngium foetidum L. & Apiaceae & Perennial & Herb & Leaf & $\begin{array}{l}\text { Added as spice in all cooked dish; } \\
\text { especially in meat curry }\end{array}$ & Year round & Common \\
\hline
\end{tabular}


Table 1 Names, Life forms, Growth habit, Edible parts, Mode of utilization, Availability period and Availability status (Continued)

\begin{tabular}{|c|c|c|c|c|c|c|c|c|}
\hline $\begin{array}{l}\text { Heiba mana } \\
\text { IBSD/WEP } 040\end{array}$ & $\begin{array}{l}\text { Exbucklandia populnea } \\
\text { (R.Br.ex Griff.)R.W.Br. }\end{array}$ & Hamamelidaceae & Perennial & Tree & $\begin{array}{l}\text { Leaf, tender } \\
\text { shoot }\end{array}$ & $\begin{array}{l}\text { Eaten raw in singju, cooked eaten } \\
\text { as vegetables or making chutney }\end{array}$ & October-April & Uncommon \\
\hline $\begin{array}{l}\text { Sita phal IBSD/WEP } \\
041\end{array}$ & Passiflora edulis Sims & Passifloraceae & Perennial & Climber & Leaf & $\begin{array}{l}\text { Cooked eaten as vegetable; added } \\
\text { to meat curry }\end{array}$ & Year round & Common \\
\hline $\begin{array}{l}\text { Torong khongnang } \\
\text { IBSD/WEP } 042\end{array}$ & Ficus benghalensis L. & Moraceae & Perennial & Tree & Bud & $\begin{array}{l}\text { Cooked eaten by boiling with chilli, } \\
\text { dry fish, peas and potato }\end{array}$ & February-March & Common \\
\hline $\begin{array}{l}\text { Pheija maton } \\
\text { IBSDNWEP } 043\end{array}$ & Wendlandia glabrata DC. & Rubiaceae & Perennial & Tree & Inflorescence & $\begin{array}{l}\text { Eaten raw as singju or with chutney } \\
\text { and also cooked eaten as eromba }\end{array}$ & $\begin{array}{l}\text { December- } \\
\text { January }\end{array}$ & Common \\
\hline $\begin{array}{l}\text { Lamthabi IBSD/WEP } \\
044\end{array}$ & Zehneria scabra Sond. & Cucurbitaceae & Annual & Climber & Leaf, fruit & $\begin{array}{l}\text { Eaten by simply boiling in water } \\
\text { with pinch of salt called champhut }\end{array}$ & July- November & Common \\
\hline $\begin{array}{l}\text { Lamthabi IBSD/WEP } \\
045\end{array}$ & Cyclanthera pedata (L.) Schrad & Cucurbitaceae & Annual & Climber & Fruit & $\begin{array}{l}\text { Eaten raw as snack, cooked eaten } \\
\text { as vegetable }\end{array}$ & $\begin{array}{l}\text { November- } \\
\text { December }\end{array}$ & Uncommon \\
\hline Singjwal IBSD/WEP 046 & Zanthoxylum budrunga DC & Rutaceae & Perennial & Tree & Leaf & $\begin{array}{l}\text { Eaten raw as singju, cooked as } \\
\text { vegetable }\end{array}$ & Year round & Common \\
\hline $\begin{array}{l}\text { Sijou mana } \\
\text { IBSD/WEP } 047\end{array}$ & Eurya acuminata DC. & Pentaphylacaceae & Perennial & Tree & Leaf & $\begin{array}{l}\text { Cooked eaten in various forms as } \\
\text { ooti, chagempomba and eromba }\end{array}$ & Year round & Common \\
\hline $\begin{array}{l}\text { Ansingteh IBSD/WEP } \\
048\end{array}$ & Lycianthes laevis (Dunal) Bitter & Solanaceae & Perennial & Herb & $\begin{array}{l}\text { Leaf, soft } \\
\text { stem }\end{array}$ & Cooked with meat or boil with rice & October-January & Common \\
\hline $\begin{array}{l}{ }^{*} \text { Anjouteh /Morok } \\
\text { maan IBSD/WEP } 049\end{array}$ & Solanum nigrum L. & Solanaceae & Annual & Herb & Leaf & $\begin{array}{l}\text { Boil and taken as such; boiled and } \\
\text { cooked with rice or meat }\end{array}$ & $\begin{array}{l}\text { November- } \\
\text { March }\end{array}$ & Common \\
\hline $\begin{array}{l}\text { Ookhamen IBSD/WEP } \\
050\end{array}$ & Solanum betaceum Cav. & Solanaceae & Perennial & Shrub & Fruit & $\begin{array}{l}\text { Cooked as vegetable or make } \\
\text { Chutney }\end{array}$ & $\begin{array}{l}\text { November- } \\
\text { January }\end{array}$ & Common \\
\hline $\begin{array}{l}\text { *Solunche/Jyan/ } \\
\text { Gariyangei } \\
\text { IBSD/WEP } 051\end{array}$ & Elatostema lineolatum Wight & Urticaceae & Perennial & Herb & $\begin{array}{l}\text { Leaf, tender } \\
\text { stem }\end{array}$ & $\begin{array}{l}\text { Cooked eaten by simply boiling or } \\
\text { with rice and other vegetables }\end{array}$ & Year round & Common \\
\hline $\begin{array}{l}\text { *Anpui /BP mana } \\
\text { IBSD/WEP } 052\end{array}$ & $\begin{array}{l}\text { Clerodendrum colebrookianum } \\
\text { Walp }\end{array}$ & Lamiaceae & Perennial & Shrub & Leaf & $\begin{array}{l}\text { Taken by boiling with salt or cooked } \\
\text { with other vegetables }\end{array}$ & Year round & Common \\
\hline $\begin{array}{l}\text { *Tharei sapou/Teravu } \\
\text { IBSD/WEP } 053\end{array}$ & Piper pedicellatum DC. & Piperaceae & Perennial & Climber & Leaf & $\begin{array}{l}\text { Taken by boiling with pinch of salt; } \\
\text { cooked as eromba or with meat }\end{array}$ & April- May & Common \\
\hline $\begin{array}{l}\text { Pfuchowbu } \\
\text { IBSD/NEP } 054\end{array}$ & $\begin{array}{l}\text { Diplazium esculentum } \\
\text { (Retz.) Sw. }\end{array}$ & Athyriaceae & Perennial & herb & Leaf & Cooked with daal & $\begin{array}{l}\text { November- } \\
\text { February }\end{array}$ & Uncommon \\
\hline Cholang IBSD/WEP 055 & Allium chinense G. Don & Amaryllidaceae & $\begin{array}{l}1-2 \\
\text { months }\end{array}$ & Herb & Whole part & $\begin{array}{l}\text { Added as spice to dish, eaten raw } \\
\text { with chutney }\end{array}$ & $\begin{array}{l}\text { December- } \\
\text { February }\end{array}$ & Common \\
\hline Huihu IBSD/WEP 056 & Derris wallichii Prain & Leguminosae & Perennial & Tree & New leaf & $\begin{array}{l}\text { Strain boiled water and cooked with } \\
\text { potato or as eromba }\end{array}$ & March-April & Uncommon \\
\hline $\begin{array}{l}\text { *Sinthupi/Galwa } \\
\text { IBSD/WEP } 057\end{array}$ & $\begin{array}{l}\text { Dysoxylum gobara } \\
\text { (Buch.-Ham) Merr. }\end{array}$ & Meliaceae & Perennial & Tree & Tender stem & $\begin{array}{l}\text { Strain boil water and cooked as } \\
\text { vegetable }\end{array}$ & March-May & Uncommon \\
\hline Chonbe IBSD/WEP 058 & Heteropanax sp. & Araliaceae & Perennial & Tree & Inflorescence & $\begin{array}{l}\text { Cooked as vegetable with dry fish } \\
\text { or meat; preparation of chutney }\end{array}$ & March & Uncommon \\
\hline Ansah IBSD/WEP 059 & $\begin{array}{l}\text { Spilanthes paniculata Wall. ex } \\
\text { DC }\end{array}$ & Asteraceae & Perennial & Herb & $\begin{array}{l}\text { Leaf, } \\
\text { inflorescence }\end{array}$ & Cooked along with other Vegetables & $\begin{array}{l}\text { Year round; } \\
\text { except summer }\end{array}$ & Common \\
\hline
\end{tabular}


Table 1 Names, Life forms, Growth habit, Edible parts, Mode of utilization, Availability period and Availability status (Continued)

\begin{tabular}{|c|c|c|c|c|c|c|c|c|}
\hline Wah-vu IBSD/WEP 060 & Polygonum molle D. Don & Polygonaceae & Perennial & Herb & Leaf & Cooked eaten as vegetable & $\begin{array}{l}\text { March- } \\
\text { November }\end{array}$ & Common \\
\hline $\begin{array}{l}\text { *Pah-vu /Yempat } \\
\text { IBSD/WEP } 061\end{array}$ & Plantago erosa Wall & Plantaginaceae & Annual & Herb & Leaf & $\begin{array}{l}\text { Cooked as eromba or along with } \\
\text { other vegetables }\end{array}$ & Year round & Common \\
\hline $\begin{array}{l}\text { Pullei manbi } \\
\text { IBSD/WEP } 062\end{array}$ & $\begin{array}{l}\text { Etlingera linguiformis } \\
\text { (Roxb.)R.M.SM }\end{array}$ & Zingiberaceae & Perennial & Herb & Rhizome & Added as an item in various dish & Year round & Common \\
\hline Laiwa IBSD/WEP063 & $\begin{array}{l}\text { Chimonobambusa callosa } \\
\text { (Munro) Nakia }\end{array}$ & Poaceae & Perennial & Shrub & New shoot & $\begin{array}{l}\text { Boiled and prepare along with chilli, } \\
\text { fermented fish, potato and pea }\end{array}$ & $\begin{array}{l}\text { September- } \\
\text { December }\end{array}$ & Common \\
\hline Naatwa IBSD/WEP 064 & $\begin{array}{l}\text { Schizostachyum munroi } \\
\text { S. Kumar \& P. Singh }\end{array}$ & Poaceae & Perennial & Shrub & New shoot & $\begin{array}{l}\text { Cooked with other vegetables and } \\
\text { meat }\end{array}$ & $\begin{array}{l}\text { November- } \\
\text { December }\end{array}$ & Common \\
\hline Gangru IBSD/WEP 065 & $\begin{array}{l}\text { Phrynium placentarium } \\
\text { (Lour.) Merr. }\end{array}$ & Marantaceae & Perennial & Herb & Rhizome & Boil and taken as such & $\begin{array}{l}\text { October - } \\
\text { November }\end{array}$ & Common \\
\hline $\begin{array}{l}\text { Anpuinu IBSD/WEP } \\
066\end{array}$ & Hiptage sp. & Malpighiaceae & Perennial & Climber & Leaf & $\begin{array}{l}\text { Eaten both raw or steam along } \\
\text { with chutney }\end{array}$ & January-June & Common \\
\hline $\begin{array}{l}\text { Moirang khanam } \\
\text { IBSD/WEP } 067\end{array}$ & $\begin{array}{l}\text { Clerodendrum serratum } \\
\text { (L.) Moon }\end{array}$ & Lamiaceae & Perennial & Shrub & $\begin{array}{l}\text { Leaf, } \\
\text { inflorescence }\end{array}$ & $\begin{array}{l}\text { Steamed and used for preparation } \\
\text { of chutney }\end{array}$ & $\begin{array}{l}\text { August- } \\
\text { September }\end{array}$ & Common \\
\hline Anthru IBSD/WEP 068 & $\begin{array}{l}\text { Momordica dioica Roxb. } \\
\text { ex Willd }\end{array}$ & Cucurbitaceae & Perennial & Climber & Tender leaf & $\begin{array}{l}\text { Cooked by boiling with rice as } \\
\text { chagempomba }\end{array}$ & Year round & Common \\
\hline
\end{tabular}

*some species have multiple names as they are known by different names in different communities 
Table 2 Criteria, weight, sub-criteria and assignment score

\begin{tabular}{|c|c|c|c|}
\hline Assignment criteria & Weight & Sub-criteria & $\begin{array}{l}\text { Assignment } \\
\text { score }\end{array}$ \\
\hline \multirow[t]{4}{*}{ C1- Taste (T) } & \multirow[t]{4}{*}{0.1934} & Most preferred & 4 \\
\hline & & Commonly preferred & 3 \\
\hline & & $\begin{array}{l}\text { Preferred but not } \\
\text { common }\end{array}$ & 2 \\
\hline & & Occasionally used & 1 \\
\hline \multirow[t]{4}{*}{ C2-Distribution (D) } & \multirow[t]{4}{*}{0.1920} & 7-9 districts & 4 \\
\hline & & 5-6 districts & 3 \\
\hline & & 3-4 districts & 2 \\
\hline & & $1-2$ districts & 1 \\
\hline \multirow[t]{3}{*}{ C3-Community status (CS) } & \multirow[t]{3}{*}{0.0749} & Dominant & 3 \\
\hline & & Common & 2 \\
\hline & & Rare & 1 \\
\hline \multirow[t]{2}{*}{ C4-Life form (LF) } & \multirow[t]{2}{*}{0.0283} & Perennial & 2 \\
\hline & & Annual/Biennial & 1 \\
\hline \multirow[t]{4}{*}{ C5-Basis of civil use (BCU) } & \multirow[t]{4}{*}{0.0576} & Wide range & 4 \\
\hline & & Commonly used & 3 \\
\hline & & Used but not common & 2 \\
\hline & & Rarely used & 1 \\
\hline \multirow[t]{2}{*}{ C6- Wild or cultivated (WC) } & \multirow[t]{2}{*}{0.0358} & Cultivated & 2 \\
\hline & & Wild & 1 \\
\hline \multirow[t]{2}{*}{ C7- Edible time (ET) } & \multirow[t]{2}{*}{0.0933} & Cross seasonal eating & 2 \\
\hline & & Single seasonal eating & 1 \\
\hline \multirow[t]{2}{*}{ C8-Edible part (EP) } & \multirow[t]{2}{*}{0.1644} & Multiple parts & 2 \\
\hline & & Single part & 1 \\
\hline \multirow[t]{2}{*}{ C9-Medicinal value (MV) } & \multirow[t]{2}{*}{0.0324} & Yes & 1 \\
\hline & & No & 0 \\
\hline \multirow[t]{3}{*}{ C10-Market potential (MP) } & \multirow[t]{3}{*}{0.1278} & High & 3 \\
\hline & & General & 2 \\
\hline & & Low & 1 \\
\hline
\end{tabular}

people of the state are traditionally dependent on the wild plant resources for various cultural and religious purposes since ancient times [39]. A large variety of such edible plants are also sold in the market as a means of livelihood for the rural population. This study highlighted the rich floral diversity and the traditional knowledge of the use of wild plants as a source of vegetable by the ethnic communities of Manipur. A total of 68 wild species belonging to 42 families have been documented and collected from the market survey. The list of plants along with their local name, life form, growth habit, use category, collection period, parts consumed, mode of consumption, availability status are presented (Table 1). Of these species, 54 (79\%) are perennial while others are annual (19\%). Their growth habit includes tree, shrub, herb, climber, creeper, weed and hydrophytes. Herbaceous plants make up the highest proportion of edible plants with 31 species (46\%), followed by trees with 15 species (22\%) and shrubs with 11 species (16\%). Among the edible parts, leaves are dominant with 33 species (49\%) followed by shoot and stem with 15 species (22\%), and most of them are consumed as cooked vegetables that include boil, steam, and fry (Fig. 2). Further, 57 species $(84 \%)$ are commonly available whereas $11(16 \%)$ are rare. As many as 51 species (75\%) are seasonal, and $17(25 \%)$ are available throughout the year. Some of them are used as herbal medicine while others are used as poultry feed, fuelwood, fencing, etc. besides their use as food. The multipurpose use of these plants can be an important reason for their conservation [40].

The communities use different modes of consumption of these species. Though the method of preparation is same, use of wild vegetables varies among communities according to their preference in taste and food habit. Singju, Eromba, Ooti, Chagempomba, Kangsoi, Champhut are the major traditional cuisines unique to the state that form an important part of daily diet. Use of one or more wild vegetables is a necessary part of a local meal. Fresh collection of vegetables before cooking is preferred.

Table 3 Judgement of matrix and consistency check of the value criteria

\begin{tabular}{|c|c|c|c|c|c|c|c|c|c|c|c|c|}
\hline \multicolumn{12}{|c|}{ Judgment matrix } & Consistency check \\
\hline & C1 & $\mathrm{C} 2$ & $\mathrm{C} 3$ & C4 & $\mathrm{C5}$ & $\mathrm{C6}$ & $\mathrm{C7}$ & $\mathrm{C} 8$ & C9 & $\mathrm{C} 10$ & Weight & \multirow{11}{*}{$\begin{array}{l}\lambda \max =11.1445 \\
C l=0.1271 \\
R I=1.49 \\
C R=0.0853<0.1\end{array}$} \\
\hline $\mathrm{C} 1$ & 1 & 2 & 3 & 3 & 2 & 5 & 5 & 3 & 5 & 3 & 0.1934 & \\
\hline $\mathrm{C} 2$ & $1 / 2$ & 1 & 3 & 3 & 2 & 3 & 5 & 3 & 5 & 3 & 0.1920 & \\
\hline $\mathrm{C} 3$ & $1 / 3$ & $1 / 3$ & 1 & $1 / 3$ & $1 / 3$ & 3 & 3 & 3 & 3 & $1 / 3$ & 0.0749 & \\
\hline $\mathrm{C} 4$ & $1 / 3$ & $1 / 3$ & 3 & 1 & $1 / 3$ & 2 & 3 & 3 & 3 & $1 / 3$ & 0.0933 & \\
\hline $\mathrm{C} 5$ & $1 / 2$ & $1 / 2$ & 3 & 3 & 1 & 5 & 5 & 3 & 3 & 3 & 0.1644 & \\
\hline C6 & $1 / 5$ & $1 / 3$ & $1 / 3$ & $1 / 2$ & $1 / 5$ & 1 & $1 / 2$ & $1 / 3$ & $1 / 3$ & $1 / 3$ & 0.0283 & \\
\hline $\mathrm{C7}$ & $1 / 5$ & $1 / 5$ & $1 / 3$ & $1 / 3$ & $1 / 5$ & 2 & 1 & $1 / 3$ & 3 & $1 / 5$ & 0.0358 & \\
\hline $\mathrm{C} 8$ & $1 / 3$ & $1 / 3$ & $1 / 3$ & $1 / 3$ & $1 / 3$ & 3 & 3 & 1 & 3 & $1 / 3$ & 0.0576 & \\
\hline C9 & $1 / 5$ & $1 / 5$ & $1 / 3$ & $1 / 3$ & $1 / 3$ & 3 & $1 / 3$ & $1 / 3$ & 1 & $1 / 3$ & 0.0324 & \\
\hline $\mathrm{C} 10$ & $1 / 3$ & $1 / 3$ & 3 & 3 & $1 / 3$ & 3 & 5 & 3 & 3 & 1 & 0.1275 & \\
\hline
\end{tabular}


Table 4 Rl value versus ' $n$ '

\begin{tabular}{|c|c|c|c|c|c|c|c|c|c|c|c|c|c|c|c|}
\hline$n$ & 1 & 2 & 3 & 4 & 5 & 6 & 7 & 8 & 9 & 10 & 11 & 12 & 13 & 14 & 15 \\
\hline $\mathrm{Rl}$ & 0.00 & 0.00 & 0.58 & 0.90 & 1.12 & 1.24 & 1.32 & 1.41 & 1.45 & 1.49 & 1.51 & 1.48 & 1.56 & 1.57 & 1.59 \\
\hline
\end{tabular}

The description of the mode of preparation of traditional dishes (Table 1) is as follows: (i) Singju, the raw dish is most common traditional food (traditional salad) made by mixing a number of wild edible species with fermented fish, chilli, and other plants (like cabbage); (ii) Eronba is prepared by boiling plant parts and smashing it with potatoes or pea, chilli, and fermented fish into a semi-liquid dish; (iii) Ootti is prepared by boiling vegetable mixture along with some rice with a pinch of sodium bicarbonate; (iv) Chagempomba is prepared by boiling soyabean, rice and different varieties of vegetables and (v) Kangsoi is a soupy dish prepared by boiling vegetable and potato with chilli, salt, fermented fish and small dried fish (see Additional file 1). Such use of WEPs in traditional delicacies was common among the tribal communities in the Himalayan Mountains [41] which explains their role in diversifying diet and fulfilling the nutritional requirement of the local system.

\section{Analysis of evaluation criteria \\ Taste, market potential and medicinal value}

Previous ethnobotanical surveys indicated that organoleptic traits can be used as the basis for value judgement and became criteria against which the value for a range of species could be evaluated [42]. For e.g. when the respondents are asked to choose which of the given two species is more significant or valuable to them, the response for one species being more valuable or significant than other is simply because it is tastier than the other. The survey participants indicated that the tastiest species are most commonly preferred by consumers, and have greater market potential though marketability is also influenced by other factors such as abundance, availability, distribution. Taste was the most important criterion for preference in case of leafy vegetables in southern Ethiopia [17], and also in Benin [43]. The taste criterion is based on the method of Jain et al. [30]. Some of the plants though not considered tasty are consumed by the locals for their medicinal quality or the health benefit they provided. Based on the survey data and literature review, 44 species $(65 \%)$ in the present study are with ethnomedicinal property. According to a report on wild vegetable resources in Inner Mongolia, 62 species of wild vegetable are used as medicine [38].

\section{Distribution, community status and life form}

The majority of the wild plants are distributed in most of the region whereas the rest are found in a certain area. As for the community status of the 68 species surveyed, 51 species $(75 \%)$ are common, 11 species (16\%) are rare, and 6 species ( $9 \%$ ) make up the dominant species. Of these species, 54 are perennial (79\%) while the rest are annual (19\%). These conditions directly influence the collection and consumption of these species.

\section{Frequency of use and whether the plant is wild or domesticated}

Based on the survey, 22 species (32\%) are widely used for frequent consumption, 42 species (61 \%) are commonly used

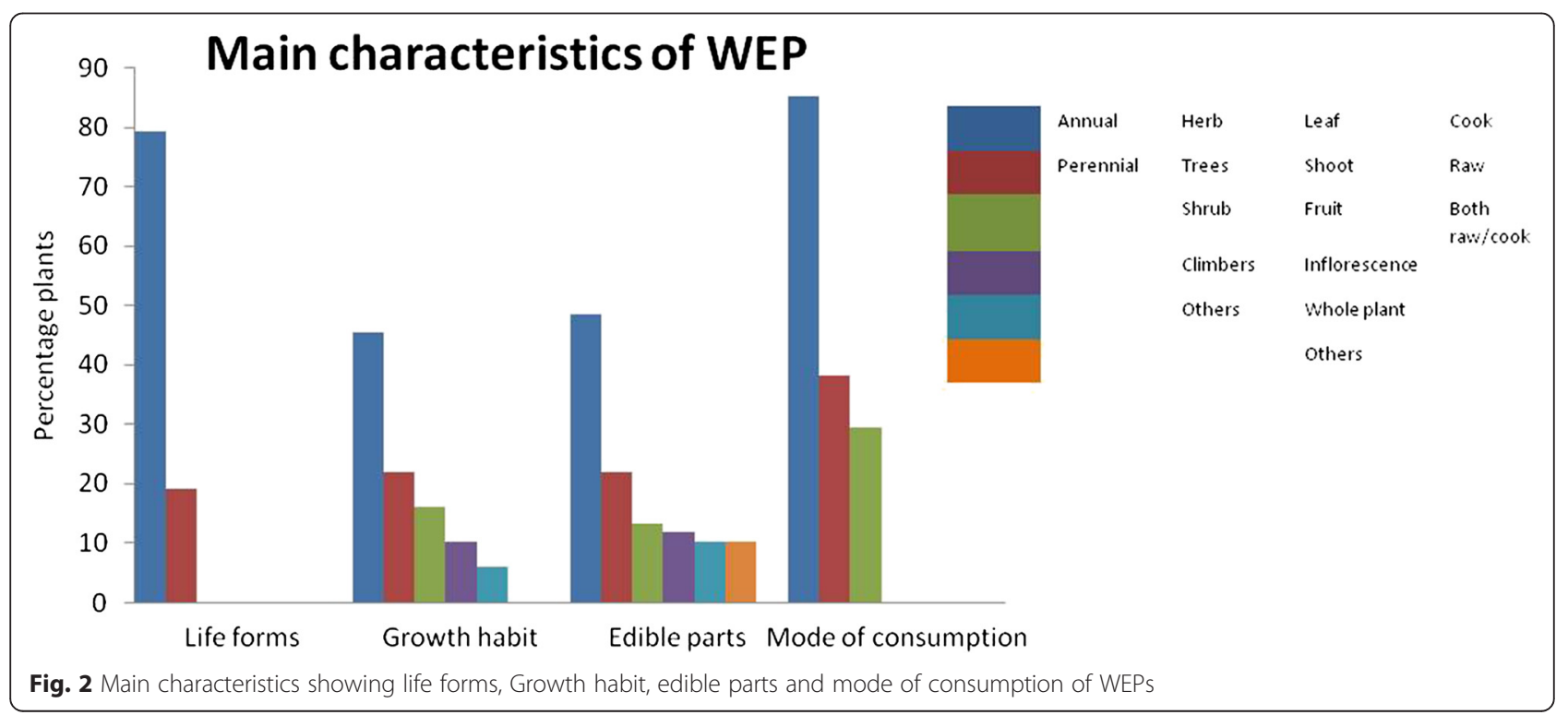


while 4 species $(6 \%)$ are rarely used. Some of the most widely used species are Euryale ferox, Chimonobambusa callosa, Ipomoea aquatica, Oenanthe javanica, Alocasia cucullata, Neptunia oleracea, Houttuynia cordata, Hedychium coronarium, Alpinia nigra, Amomum aromaticum, Eryngium foetidum, Passiflora edulis, Ficus benghalensis, Zanthoxylum budranga. It could be attributed to their taste, availability in multiple seasons or high abundance in its season and their use in various cuisines. Of the 68 species, 31 species (46\%) are semi-domesticated. They have been put into cultivation practice especially in kitchen garden while the remaining species are found in the wild. It is observed that people focus on planting those species that have good market value, taste and consumed more frequently. Usually, the tastiest species also score high concerning marketability (based on survey). Kidane et al. [17] emphasized the importance of home gardens for promotion and cultivation of prioritized leafy vegetables for ease of management, ownership, supervision and intensive cultural practices in cultivated land.

\section{Edible parts and edible time}

Sixteen species (24\%) are consumed for more than one part of the plant whereas 52 species $(76 \%)$ are collected for their single part. The edible parts include leaf, stem, fruit, root, rhizome, bud, tuber and inflorescence. Among them, leaves are dominant followed by shoot and stem and most of these are consumed as cooked vegetables. Consumption of 40 species (59\%) is single seasonal, and 28 species (41\%) are used in multiple seasons.

\section{Integrated value}

According to the integrated value (Table 5), the wild vegetables in Manipur can be classified into 4 categories (Fig. 3) - highest (integrated value > 2.5), high (integrated value 2.0 - 2.5), general (integrated value1.5 2.0 ) and low (integrated value $<1.5$ ). There are only 2 species with the highest value, 26 species with high value, 31 species with general value and 9 species with low value. Overall, 57 species (84 \%) have a high or general value. Some high scoring vegetables include Centella asiatica, Euryale ferox, Chimonobambusa callosa, Ipomoea aquatica, Alocasia cucullata, Neptunia oleracea, Hedychium coronarium, Eryngium foetidum, Ficus benghalensis, Cycus pectinata, Cissus javanica, Wendlandia glabrata, and Elatostema lineolatum. It could be due to their traits of high-quality vegetables such as unique taste, appropriate edible parts, high abundance in its season, ease of

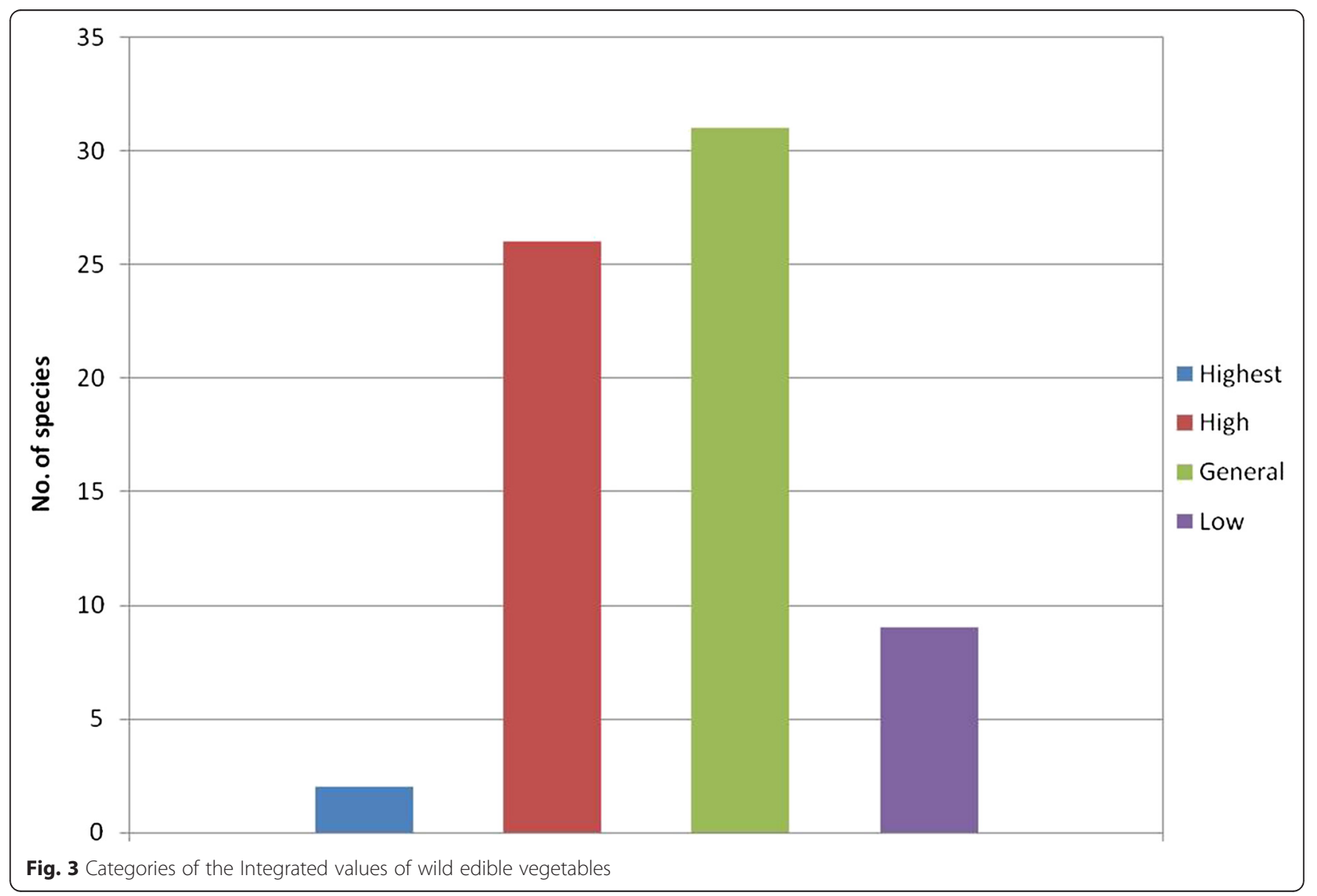


Table 5 Integrated values of evaluation criteria of the wild edible vegetables of Manipur

\begin{tabular}{|c|c|c|c|c|c|c|c|c|c|c|c|c|}
\hline Local names & Scientific names & $\mathrm{T}$ & $\mathrm{D}$ & CS & LF & $\mathrm{BCU}$ & WC & ET & EP & MV & MP & IV \\
\hline Chuchurangmei & Sesbania sesban (L.) Merr. & 3 & 2 & 2 & 1 & 2 & 1 & 1 & 2 & 1 & 1 & 1.87 \\
\hline Kolamni & Ipomoea aquatica Forssk. & 3 & 2 & 3 & 2 & 3 & 1 & 2 & 1 & 1 & 2 & 2.09 \\
\hline Komprek & Oenanthe javanica (Blume) DC. & 3 & 3 & 2 & 2 & 3 & 1 & 2 & 1 & 1 & 1 & 2.08 \\
\hline Sinjupaal & Alocasia cucullata (Lour.) G. Don & 4 & 2 & 3 & 2 & 3 & 2 & 2 & 1 & 0 & 3 & 2.41 \\
\hline Yelang & Polygonum barbatum L. & 3 & 2 & 2 & 1 & 2 & 2 & 1 & 1 & 1 & 1 & 1.74 \\
\hline Kakthum & Eleocharis dulcis (Burm. f.) Trin. ex Hensch & 3 & 2 & 2 & 1 & 1 & 1 & 1 & 1 & 0 & 2 & 1.74 \\
\hline Ching yensil & Antidesma diandrum (Roxb.)B. Heyne. ex Roth & 3 & 2 & 2 & 2 & 2 & 1 & 2 & 1 & 1 & 2 & 1.96 \\
\hline Kengoi & Lysimachia ovovata Buch.-Ham. ex Wall & 3 & 2 & 2 & 1 & 2 & 2 & 1 & 1 & 1 & 1 & 1.74 \\
\hline Peruk & Centella asiatica (L.) Urb. & 4 & 3 & 3 & 2 & 4 & 2 & 2 & 2 & 1 & 2 & 2.73 \\
\hline Thambou & Nelumbo nucifera Gaertn. & 3 & 2 & 2 & 2 & 2 & 1 & 2 & 2 & 1 & 3 & 2.25 \\
\hline Tharo & Nympheae nouchali Burm. f. & 2 & 2 & 2 & 2 & 1 & 1 & 1 & 2 & 1 & 1 & 1.75 \\
\hline Thangjing & Euryale ferox Salisb. & 4 & 2 & 2 & 1 & 4 & 2 & 1 & 1 & 1 & 3 & 2.30 \\
\hline Esing ekaithabi & Neptunia oleracea Lour. & 4 & 2 & 2 & 1 & 3 & 2 & 1 & 1 & 1 & 3 & 2.25 \\
\hline Koukha & Sagittaria sagittifolia $\mathrm{L}$. & 3 & 2 & 2 & 1 & 2 & 1 & 1 & 1 & 1 & 2 & 1.83 \\
\hline Yendang & Cycas pectinata Buch.-Ham & 4 & 2 & 1 & 2 & 2 & 1 & 1 & 1 & 1 & 3 & 2.11 \\
\hline Monsaobi & Chenopodium album L. & 2 & 2 & 2 & 1 & 2 & 1 & 1 & 1 & 1 & 1 & 1.51 \\
\hline Kanghumaan & Meriandra bengalensis (Roxb.) Benth. & 1 & 2 & 1 & 2 & 1 & 2 & 2 & 1 & 1 & 2 & 1.47 \\
\hline Tekta & Pogostemon purpurascens Dalzell & 3 & 2 & 1 & 1 & 2 & 2 & 1 & 1 & 0 & 2 & 1.76 \\
\hline Yerum keirum & Stellaria media (L.) Vill. & 3 & 2 & 2 & 1 & 2 & 1 & 1 & 2 & 1 & 1 & 1.87 \\
\hline Toninkhok & Houttuynia cordata Thunb. & 3 & 3 & 3 & 2 & 3 & 2 & 2 & 2 & 1 & 2 & 2.48 \\
\hline Loklei & Hedychium coronarium J. Koenig & 4 & 3 & 2 & 2 & 3 & 2 & 1 & 1 & 1 & 3 & 2.47 \\
\hline Pullei & Alpinia nigra (Gaertn.) Burtt & 3 & 3 & 2 & 2 & 3 & 2 & 1 & 1 & 1 & 2 & 2.15 \\
\hline Namra & Amomum aromaticum Roxb. & 3 & 3 & 2 & 2 & 3 & 2 & 1 & 1 & 1 & 2 & 2.15 \\
\hline Yaipal & Curcuma angustifolia Roxb. & 2 & 2 & 2 & 2 & 2 & 2 & 1 & 1 & 1 & 2 & 1.7 \\
\hline Sarei mapan & Amomum sp. & 3 & 2 & 1 & 2 & 2 & 1 & 1 & 1 & 0 & 2 & 1.76 \\
\hline Esing kambong & Zizania latifolia (Griseb.) Turcz. ex Stapf & 4 & 2 & 1 & 2 & 2 & 1 & 1 & 1 & 1 & 3 & 2.11 \\
\hline Chantruk mana & Cardamine hirsute $\mathrm{L}$. & 2 & 2 & 2 & 1 & 2 & 2 & 2 & 1 & 1 & 2 & 1.76 \\
\hline Huikhong/ Mansam & Viola pilosa Blume & 3 & 2 & 2 & 2 & 2 & 1 & 2 & 2 & 1 & 2 & 2.12 \\
\hline Phunil & Gnaphalium indicum L. & 2 & 2 & 2 & 1 & 2 & 2 & 1 & 2 & 1 & 2 & 1.83 \\
\hline Kongouyen & Cissus javanica DC & 3 & 3 & 2 & 2 & 2 & 1 & 2 & 1 & 1 & 2 & 2.12 \\
\hline Heibi mana & Vangueria spinosa (Roxb. ex Link) Roxb. & 2 & 2 & 2 & 2 & 2 & 2 & 1 & 1 & 0 & 2 & 1.64 \\
\hline Lam khamen & Solanum torvum Sw. & 1 & 2 & 2 & 2 & 2 & 1 & 1 & 1 & 0 & 1 & 1.32 \\
\hline Nongmangkha & Phlogacanthus thyrsiformis (Roxb.ex Hardw.) Mabb & 3 & 3 & 2 & 2 & 2 & 2 & 1 & 2 & 1 & 2 & 2.25 \\
\hline Oosingsha mapaan & Litsea cubeba Pers. & 2 & 3 & 2 & 2 & 2 & 1 & 1 & 2 & 1 & 2 & 2.02 \\
\hline Chigonglei angouba & Leucaena leucocephala(Lam.) de Wit & 2 & 2 & 2 & 2 & 2 & 2 & 1 & 1 & 1 & 2 & 1.9 \\
\hline Oothum maton & Wendlandia paniculata (Roxb.) DC. & 2 & 3 & 2 & 2 & 2 & 2 & 1 & 1 & 0 & 2 & 1.67 \\
\hline Mukthrubi & Zanthoxylum acanthopodium DC. & 3 & 3 & 2 & 2 & 2 & 2 & 2 & 1 & 1 & 2 & 2.18 \\
\hline Naoseknambi / Anpajul & Zanthoxylum sp. & 3 & 1 & 2 & 2 & 2 & 2 & 1 & 1 & 0 & 2 & 1.67 \\
\hline Awaphadigom & Eryngium foetidum $\mathrm{L}$. & 4 & 3 & 2 & 2 & 3 & 2 & 2 & 1 & 1 & 3 & 2.56 \\
\hline Heiba mana & Exbucklandia populnea (R.Br. ex. Griff.) R.W. Br. & 3 & 2 & 2 & 2 & 2 & 2 & 2 & 1 & 0 & 2 & 1.96 \\
\hline Sitaphal mana & Passiflora edulis Sims & 3 & 3 & 2 & 2 & 3 & 2 & 2 & 2 & 1 & 2 & 2.41 \\
\hline Torong khongnang & Ficus benghalensis L. & 4 & 3 & 2 & 2 & 3 & 1 & 1 & 2 & 0 & 2 & 2.43 \\
\hline Pheija mapan & Wendlandia glabrata DC. & 3 & 3 & 2 & 2 & 3 & 1 & 1 & 1 & 1 & 2 & 2.11 \\
\hline Lamthabi mana & Zehneria scabra Sond. & 3 & 1 & 1 & 2 & 2 & 1 & 1 & 1 & 0 & 2 & 1.56 \\
\hline
\end{tabular}


Table 5 Integrated values of evaluation criteria of the wild edible vegetables of Manipur (Continued)

\begin{tabular}{|c|c|c|c|c|c|c|c|c|c|c|c|c|}
\hline Lamthabi & Cyclanthera pedata (L.) Schrad. & 2 & 1 & 1 & 2 & 2 & 1 & 1 & 2 & 1 & 3 & 1.69 \\
\hline Singjwal & Zanthoxylum budrunga DC & 4 & 1 & 3 & 2 & 3 & 1 & 2 & 1 & 0 & 2 & 2.05 \\
\hline Sijou mana & Eurya acuminata DC. & 3 & 1 & 3 & 2 & 3 & 2 & 2 & 1 & 0 & 1 & 1.77 \\
\hline Ansingteh & Lycianthes laevis (Dunal) Bitter & 2 & 1 & 2 & 2 & 2 & 1 & 1 & 1 & 0 & 2 & 1.44 \\
\hline Anjouteh/Morokmaan & Solanum nigrum L. & 2 & 3 & 2 & 1 & 2 & 1 & 2 & 1 & 1 & 2 & 1.92 \\
\hline Ookhamen & Solanum betaceum Cav. & 2 & 2 & 1 & 2 & 2 & 2 & 2 & 1 & 1 & 2 & 1.72 \\
\hline Solunche/Jyan/Gariyangei & Elatostema lineolatum Wight & 4 & 2 & 2 & 2 & 3 & 1 & 2 & 2 & 0 & 2 & 2.34 \\
\hline Anpui/Bp mana & Clerodendrum colebrookianum Walp & 3 & 2 & 2 & 2 & 3 & 1 & 2 & 1 & 1 & 2 & 2.01 \\
\hline Tharei sapou/Teravu/Thimnahan & Piper pedicellatum C. DC. & 3 & 1 & 2 & 2 & 3 & 1 & 2 & 1 & 1 & 2 & 1.81 \\
\hline Pfuchowbu & Diplazium esculentum (Retz.) Sw & 2 & 1 & 2 & 2 & 2 & 2 & 1 & 1 & 0 & 2 & 1.48 \\
\hline Cholang & Allium chinense G. Don & 2 & 2 & 2 & 1 & 2 & 2 & 2 & 2 & 1 & 2 & 1.93 \\
\hline Huihu & Derris wallichii Prain & 3 & 1 & 1 & 2 & 2 & 1 & 2 & 1 & 0 & 2 & 1.66 \\
\hline Sinthupi/Galwa & Dysoxylum gobara (Buch.-Ham) Merr & 2 & 1 & 1 & 2 & 2 & 1 & 1 & 1 & 0 & 3 & 1.49 \\
\hline Chonbe & Heteropanax sp. & 3 & 1 & 2 & 2 & 3 & 2 & 1 & 1 & 0 & 3 & 1.86 \\
\hline Ansah & Spilanthes paniculata Wall. ex DC & 2 & 3 & 2 & 2 & 2 & 1 & 2 & 2 & 1 & 2 & 2.12 \\
\hline Wah-vu & Polygonum molle D. Don & 2 & 1 & 2 & 2 & 2 & 1 & 1 & 1 & 0 & 2 & 1.44 \\
\hline Pah-vu/yempat & Plantago erosa Wall & 3 & 3 & 2 & 2 & 3 & 1 & 1 & 1 & 1 & 2 & 2.11 \\
\hline Pullei manbi & Etlingera linguiformis (Roxb.) R.M. Sm. & 3 & 2 & 2 & 2 & 2 & 2 & 2 & 1 & 1 & 2 & 1.99 \\
\hline Laiwa & Chimonobambusa callosa (Munro) Nakia & 4 & 2 & 2 & 1 & 4 & 1 & 1 & 1 & 0 & 3 & 2.24 \\
\hline Naatwa & Schizostachyum munroi & 2 & 2 & 2 & 2 & 1 & 1 & 1 & 1 & 0 & 2 & 1.58 \\
\hline Gangru & Phrynium placentarium (Lour.) Merr. & 3 & 1 & 1 & 2 & 2 & 2 & 1 & 1 & 0 & 2 & 1.6 \\
\hline Anpuinu & Hiptage sp. & 2 & 1 & 2 & 2 & 2 & 1 & 1 & 1 & 1 & 1 & 1.35 \\
\hline Moirang khanam & Clerodendrum serratum (L.) Moon & 1 & 2 & 2 & 2 & 2 & 1 & 2 & 1 & 1 & 1 & 1.44 \\
\hline Anthru & Momordica dioica Roxb.ex Willd. & 2 & 1 & 2 & 2 & 2 & 1 & 2 & 1 & 0 & 1 & 1.41 \\
\hline
\end{tabular}

processing, high market value and so on. They are also among the most preferred and frequently consumed species.

An integrated assessment of wild species has not yet been done in Manipur and elsewhere except Inner Mongolia, China [38]. It provides scientific clues to select priority and high-quality species. The present study developed a new approach to the integrated assessment of wild leafy vegetables based on a set of defined criteria. The result highlighted that 57 species (84\%) have good to high value (Table 5). Among the high scoring species, Zanthoxylum budrunga, Passiflora edulis, Clerodendrum colebrookianum, Spilanthes paniculata, Cissus javanica, Elatostema lineolatum, Plantago erosa, Litsea cubeba, etc. and other species such as Zehneria scabra, Cyclanthera pedata, Piper pedicellatum, Solanum nigrum, Eurya acuminate, Solanum betaceum, Allium chinense, Heteropanax sp., Dysoxylum gobara, Diplanzium esculantum, Etlingera linguiformis, Derris wallichii, Phrynium placentarium are found to be consumed mainly by the tribal communities and rarely known to other communities. It may be due to their traditional food habit experience, preference, and local species availability. Many more such unexplored leafy vegetables are believed to exist. There is a need for exploitation of such unexplored resources given the storehouse of traditional knowledge the tribal possessed. It will provide a way for screening newer and alternative source of nutrition.

The present finding will be useful in the evaluation of nutritional components of high priority species for their integration into the agricultural system based on nutritive values. Further, assessing their cultivable potential and working towards developing agro-techniques can bring more potential species under domestication for conservation through sustainable use. Moreover, it will also help to understand their role in future food and nutritional security of the state.Therefore, documentation and prioritization would ensure that the highest priority species is preserved for use in crop improvement programs and contribute towards achieving the goal of food and nutritional security.

\section{Traditional knowledge (TK)}

WEPs constitute an integral part of the indigenous socioecological system associated with traditional ecological knowledge of ethnic communities. We observed that 
women ( $>40$ years old) of a household possessed more traditional knowledge about leafy vegetables including the identity of the species, usage, and mode of preparation. It could be due to their association with household chores such as cooking, marketing, and their home nurturing qualities. Upetry et al. [18] have reported a similar finding. Phillips and Gentry [44] also reported that WEP knowledge is gained early in life and increases with age.

Participants in the survey have mentioned a declined in the traditional knowledge of natural resources in recent times. The cultural and traditional association of WEPs with the ethnic communities is gradually falling as they are not passed down to future generations, so present generations have little information regarding wild edibles associated with the diet of their ancestors. These generations are no longer interested in acquiring traditional knowledge of WEPs. Presumably, increasing level of modernization significantly contributes to the erosion of TK. Benz et al. [45] explained the abandoning of aboriginal ancestral practices by indigenous people in Mexico for economic and social gain. Loss of knowledge may occur if resources disappear from the landscape. Srivastava and Singh [46] have reported that frequent and overexploitation of species leads to threat in survival for some species of Northeastern States. However, the loss of indigenous knowledge worldwide has varied reasons and has been explained under local, ecological, socio-economic and cultural contexts [17]. Studying major grounds for the decline of resources and loss of associated knowledge will help decision makers in their formulations and analysis of policy [47]. Documentation and evaluation of traditional knowledge related to diversity, usage, and status of WEPs are crucial for preserving it for future generations. Support of TK systems and empowerment of its knowledge holders, promotion of the use of TK, recognition of rights of TK holders relating to their knowledge are the bottom-up approach to development [48]. It should be supported by complementary in-situ an ex-situ conservation strategies to conserve and sustainably utilize the natural resources and associated knowledge.

\section{Conclusion}

The diverse use of wild plant resources for food, medicine, income and socio-cultural purposes by the ethnic communities of Manipur revealed the high dependence on them with as many as 68 wild vegetables documented and collected. Though Manipur is bountiful in wild vegetable, a large number of them remain unexplored or known to certain sections of society. Traits that contribute to the uncommon usage of these plants include different food habits and experience of ethnic communities, the difference in taste preference, distribution, abundance and edibility time.

According to the integrated assessment, 57 out of 68 (84 \%) species have good to high value. These high scoring species exhibit the traits of high-quality vegetables, such as taste, appropriate edible parts, multiple edible parts, availability, abundance, easily cultivable, simple to collect and process, and so on. To increase dietary diversity and livelihood sustenance of local people, complimentary studies and further ethnobotanical studies will be conducted. The traditional knowledge and understanding of wild food plants may serve as baseline data for future research and development activities and further biotechnological intervention. A detailed evaluation of nutritional components of the potential species should be conducted for integration into the agricultural system based on their nutritive values and for the conservation of elite germplasm. Further studies should also be done to assess their cultivable potential and work towards developing propagation and agro-techniques to bring more potential wild species under domestication for sustainable utilization of natural resources. Furthermore, proper value chain development for marketing and value-addition of selected species can facilitate enough income to native communities. Documentation and conservation of highest priority species would ensure they are available for use in genetic improvements of crop species as a contribution towards food and nutritional security. Therefore, communities should engage in sustainable management and preservation of traditional knowledge of these multi-valued resources for the well-being local communities.

\section{Additional file}

Additional file 1: Major cuisines of Manipur. (PDF $481 \mathrm{~kb}$ )

Competing interests

The authors declared that they had no competing interests.

Author's contribution

BT supervised the research work. SK conducted field surveys and interviews with the local vendors; drafted and finalized the manuscript with BT and $\mathrm{AKH}$. The authors have read and approved the final manuscript.

\section{Author's information}

BT, Plant Taxonomist, Scientist C, IBSD, Imphal, DBT, GOI. KS, Research

Scholar, IBSD, Imphal. AKH, Professor, Department of Biotechnology, Gauhati University, Guwahati.

\section{Acknowledgements}

The study was supported by Department of Biotechnology, Govt. of India. The authors thank the Director of IBSD for providing facilities for conducting the study. We would like to thank Dr.DK Hore for his valuable advice. We are also thankful to Dr. A Chattoraj for his help in checking the manuscript. We also acknowledge the local people and market vendors of Manipur for providing vital information during the period of the study. Our appreciation goes to the staff of PSCL, IBSD for their support and guidance.

\section{Author details}

${ }^{1}$ Plant Systematics and Conservation Laboratory (PSCL), Institute of

Bioresources and Sustainable Development (IBSD), Takyelpat, Imphal 795001, Manipur, India. ²Department of Biotechnology, Gauhati University, Guwahati 781014, India. 
Received: 19 June 2015 Accepted: 19 January 2016

\section{-}

\section{References}

1. Misra S, Maikhuri RK, Kala CP, Rao KS, Saxena KG. Wild leafy vegetables: A study of their subsistence dietetic support to the inhabitants of Nanda Devi Biosphere Reserve. India J of Ethnobiol and Ethnomed. 2008:4:15.

2. FAO. Traditional Food Plants: A Resource Book for Promoting the Exploitation and Consumption of Food plants in Arid, semi arid and Sub humid Lands of Eastern Africa, Food and nutrition paper 4-2. Rome: FAO; 1988.

3. Singh HB, Arora RK. Wild edible plants of India. 1st ed. New Delhi: ICAR Publication; 1978.

4. Schippmann $U$, Cunningham $A B$, Leaman DJ. Impact of cultivation and gathering of medicinal plants on biodiversity: Global trends and issues in Biodiversity and the Ecosystem Approach in Agriculture, Forestry and Fisheries. Rome: FAO; 2002.

5. Gemedo-Dalle TB, Maass L, Isselstein J. Plant biodiversity and ethnobotany of Borana pastoralists in southern Oromla. Ethiopia Eco Bot. 2005:59:43-65.

6. Shrestha PM, Dhillion SS. Diversity and traditional knowledge concerning wild food species in a locally managed forest in Nepal. Agroforest Syst. 2006;66:55-63.

7. Teklehaymanot T, Giday M. Ethnobotanical study of wild edible plants of Kara and Kwego semi-pastoralist people in Lower Omo River Valley, Debub Omo Zone. SNNPR Ethiopia J Ethnobiol Ethnomed. 2010;6:23.

8. Ogle BM, Grivetti LE. Legacy of the chameleon edible wild plants in the Kingdom of Swaziland, South Africa. A cultural, ecological, nutritional study. Parts II-IV, species availability and dietary use, analysis by ecological zone. Ecol Food Nutr. 1985:17:1-30.

9. Ali-Shtayeh MS, Jamous RM, Al-Shafie JH, Elgharabah WA, Kherfan FA, Qarariah $\mathrm{KH}$, et al. Traditional knowledge of wild edible plants used in Palestine (Northern West Bank): a comparative study. J Ethnobiol Ethnomed. 2008;4:13.

10. Balemie K, Kebebew F. Ethnobotanical study of wild edible plants in Derashe and Kucha Districts. South Ethiopia J Ethnobiol Ethnomed. 2006:2:53.

11. Sanchez-Mata MC, Cabrera Loera RD, Morales P, Fernandez-Ruiz V, Cámara M, Díez Marqués C, et al. Wild vegetables of the Mediterranean area as valuable sources of bioactive compounds. Gen Resour Crop Evol. 2012;59:431-43.

12. Pereira C, Barros L, Carvalho AM, Ferreira ICFR. Nutritional composition and bioactive properties of commonly consumed wild greens: Potential sources for new trends in modern diets. Food Res Int. 2011:44:2634-40.

13. Nordeide MB, Hatloy A, Folling M, Lied E, Oshoug A. Nutrient Composition and nutritional importance of green leaves and wild foods in an agricultural district, Koutiala, in Southern Mali. Int J Food Sci Nutr. 1996;47(6):455-68.

14. Sundriyal M, Sundriyal RC. Wild edible plants of the Sikkim Himalaya: Nutritive values of selected species. Eco Bot. 2001;55(3):377-90.

15. Hajjar R, Hodgkin T. The use of wild relatives in crop improvement: a survey of developments over the last 20 years. Euphytica. 2007;156:1-13.

16. Pandey A, Tomer AK, Bhandari DC, Pareek SK. Towards collection of wild relatives of crop plants in India. Gen Resour Crop Evol. 2007:55:187-202.

17. Kidane B, Van der Maesen LJG, Asfaw Z, Sosef MSM, Andel VT. Wild and semi-wild leafy vegetables used by the Maale and Ari ethnic communities in southern Ethiopia. Gen Resour Crop Evol. 2015;62(2):221-34.

18. Uprety Y, Poudel CR, Shrestha KK, Rajbhandary S, Tiwari NN, Shrestha UB, et al. Diversity of use and local knowledge of wild edible plant resources in Nepal. J Ethnobiol Ethnomed. 2012:8:16.

19. Sansanelli S, Tassoni A. Wild food plants traditionally consumed in the area of Bologna (Emilia Romagna region, Italy). J of Ethnobiol Ethnomed. 2014; 10:69

20. Strymets N, Elbakidze M, Ceuterick M, Angelstam P, Axelsson R. From economic survival to recreation: contemporary uses of wild food and medicine in rural Sweden. Ukraine and NW Russia J of Ethnobiol Ethnomed. 2015;11:53.

21. Jain AK, Tiwari P. Nutritional value of some traditional edible plants used by tribal communities during emergency with reference to central India. Indian J Tradi Knowl. 2012;11(1):51-7.

22. Rasingam L: Ethnobotanical studies on the wild edible plants Irula tribes of Pillur Valley, Coimbatore district, Tamil Nadu, India. Asian Pacific Journal of Tropical Biomedicine. 2012; 1493-97.

23. Jadhav VD, Mahadkar SD, Valvi SR. Documentation and ethnobotanical survey of wild edible plants from Kolhapur district. Recent Res Sci Technol. 2011;3(12):58-63.

24. Angami A, Gajurel PR, Rethy P, Singh B, Kalita SK. Status and potential of wild edible plants of Arunachal Pradesh. Indian J Tradi knowl. 2012;11(1):51-7.

25. Sundriyal M, Sundriyal RC. Underutilized edible plants of Sikkim Himalaya: Need for domestication. Curr Sci. 2003;85(6):731-6.
26. Pemberton RW, Lee NS. Wild food plants in South Korea; market presence, new crops, and exports to the United States. Eco Bot. 1996;50(1):57-70.

27. Luczaj L, Koncic MZ, Milicevic T, Dolina K, Pandza M. Wild vegetable mixes sold in the local markets of Dalmatia (Southern Croatia). J Ethnobiol Ethnomed. 2013;9:2

28. Dogan Y, Ugulu I, Durkan N. Wild edible plants sold in the local markets of Izmir. Turkey Pak J Bot. 2013;45(S1):177-84.

29. Bye RA, Linares $E$. The role of plants in the Mexican markets and their importance in ethnobotanical studies. J Ethnobiol. 1983;3(1):1-15.

30. Jain A, Sundriyal M, Roshnibala S, Kotoky R, Kanjilal PB, Singh HB, et al. Dietary Use and Conservation Concern of Edible Wetland Plants at Indo-Burma Hotspot: A Case Study from Northeast India. J Ethnobiol Ethnomed. 2011;7:29.

31. Gangte HE, Thoudam NS, Zomi GT. Wild Edible Plants used by Zou Tribe in Manipur. India International Journal of Scientific and Research. 2013;3:5.

32. Phoze NL, Kumar Y, Myrboh B. Survey and assessment of floral diversity on wild edible plants from Senapati district of Manipur, Northeast India. JBio EnvSci. 2001;1(6):50-62.

33. Ghose GK. Herbs of Manipur. New Delhi: A.P.H. Publishing Corporation, New Delhi; 2000.

34. BSI: In Flora of Manipur. Volume 1.Botanical Survey of India (BSI), Kolkata, India; 2000.

35. The Plant List website: http://www.theplantlist.org/ (2013). Accessed 30 September 2015.

36. Saaty TL. The Analytic Hierarchy Process. New York: McGraw-Hill; 1980.

37. Saaty TL. Fundamentals of the Analytical Hierarchy Process. 4922 Ellsworth Avenue, Pittsburg: RWS Publication; 2000

38. Wujisguleng W, Khasbagen K. An integrated assessment of wild vegetable resources in Inner Mongolian Autonomous Region. China J Ethnobiol Ethnomed. 2010;6:34.

39. Singh HB, Puni $L$, Jain A, Singh RS, Rao PG. Status, utility threats and conservation options for rattan resources in Manipur. Curr Sci. 2004;87:90-4.

40. Etkin NL. Local Knowledge of Biotic Diversity and its Conservation in Rural Hausaland. Northern Nigeria Eco Bot. 2002:56(1):73-88.

41. Sundriyal $M$, Sundriyal RC, Sharma E. Dietary use of wild plant resources in the Sikkim Himalaya. India Eco Bot. 2004:58(4):626-38.

42. N'danikou S, Achigan-Dako EG, Wong JLG. Eliciting local values of wild edible plants in Southern Bénin to identify priority species for conservation. Eco Bot. 2011;65(4):381-95.

43. Dansi A, Adjatin A, Adoukonou-Sagbadja H, Falade V. YedomonhanH, Odou D, Dossou B: Traditional leafy vegetables and their use in the Benin Republic. Gen Resour Crop Evol. 2008;55:1239-56.

44. Phillips O, Gentry AH. The useful plants of Tamboapata, Peru: II additional hypothesis testing in quantitative ethnobotany. Eco Bot. 1993:47:33-43.

45. Benz BF, Cevallos J, Santana F, Rosales J, Graf S. Losing knowledge about plant use in the Sierra de Manantlan biosphere reserve. Mexico Eco Bot. 2000:54(2):183-91.

46. Srivastava RC, Singh RK, Mukherjee TK. Bioculturally important rare new plant species of Heteropanax Seems (Araliaceae) from Eastern Himalaya, Arunachal Pradesh. Indian J Tradi Knowle. 2010;9(2):242-24.

47. Hadjichambis AC, Paraskeva-Hadjichambi D, Della A, Giusti ME, De Pasquale C, Lenzarini C, et al. Wild and semi-domesticated food plant consumption in seven circum-Mediterranean areas. Int J Food Sci Nutr. 2008;59(5):383-414.

48. WIPO: Intellectual property and traditional knowledge. Booklet no. 2. http://www.wipo.int/edocs/pubdocs/en/tk/920/wipo_pub_920.pdf. Accessed 5 October 2015

\section{Submit your next manuscript to BioMed Central and we will help you at every step:}

- We accept pre-submission inquiries

- Our selector tool helps you to find the most relevant journal

- We provide round the clock customer support

- Convenient online submission

- Thorough peer review

- Inclusion in PubMed and all major indexing services

- Maximum visibility for your research

Submit your manuscript at www.biomedcentral.com/submit 